\title{
Some applications of Wagner's weighted subgraph counting polynomial
}

\author{
Ferenc Bencs* \\ Alfréd Rényi Institute of Mathematics \\ Budapest, Hungary \\ ferenc. bencs@gmail.com
}

\author{
Péter Csikvári ${ }^{\dagger}$ \\ Alfréd Rényi Institute of Mathematics and \\ Department of Computer Science \\ Eötvös Loránd University \\ Budapest, Hungary \\ peter.csikvari@gmail.com
}

\author{
Guus Regts $\ddagger$ \\ Korteweg de Vries Institute for Mathematics \\ University of Amsterdam \\ Amsterdam, The Netherlands \\ guusregts@gmail.com
}

Submitted: Jan 22, 2021; Accepted: Jul 27, 2021; Published: Oct 22, 2021

(C) The authors. Released under the CC BY license (International 4.0).

\begin{abstract}
We use Wagner's weighted subgraph counting polynomial to prove that the partition function of the anti-ferromagnetic Ising model on line graphs is real rooted and to prove that roots of the edge cover polynomial have absolute value at most 4 . We more generally show that roots of the edge cover polynomial of a $k$-uniform hypergraph have absolute value at most $2^{k}$, and discuss applications of this to the roots of domination polynomials of graphs. We moreover discuss how our results relate to efficient algorithms for approximately computing evaluations of these polynomials.
\end{abstract}

Mathematics Subject Classifications: Primary: 05C35. Secondary: 05C31, 05C70, 05C 80

${ }^{*}$ Supported by the NKFIH (National Research, Development and Innovation Office, Hungary) grant KKP-133921.

${ }^{\dagger}$ Supported by the Counting in Sparse Graphs Lendület Research Group.

${ }_{\ddagger}^{\ddagger}$ Supported by a NWO Vidi grant, VI.Vidi.193.068 


\section{Introduction}

The investigation of the location of zeros of different partition functions of graphs and hypergraphs is a topic gaining more and more interest. The reason for this is that these partition functions are related to several topics such as statistical physics, combinatorics and computer science. In statistical physics absence of complex zeros near the real axis implies absence of phase transition (in the Lee-Yang sense [28]). In computer science it is related to the design of efficient approximation algorithms for computing evaluations of partition functions and graph polynomials. A recent approach by Barvinok [4] combined with results from [24] shows that zero-free regions for graph polynomials imply fast (polynomial time) algorithms for approximating evaluations when restricted to bounded degree graphs.

In this note we give two new zero-free regions, one for the anti-ferromagnetic Ising model on line graphs, and one for the edge cover polynomial. For both these polynomials efficient approximation algorithms were known on the positive real line. For the Ising model this was based on the Monte Carlo Markov chain approach [13], and for the edge cover polynomial on correlation decay [20, 21] as well as on the Monte Carlo Markov chain approach $[6,17]$. Our results yield new efficient algorithms for these polynomials on bounded degree graphs, not only for evaluations on the positive real line, but also for complex evaluations. More importantly they further stress the connection between absence of zeros and the existence of efficient algorithms.

Both our results are based on two short applications of a general technique of Wagner [27].

\section{The Ising model on line graphs}

Our first example is the Ising model of line graphs. Let $G=(V, E)$ denote a simple graph and let $z, b \in \mathbb{C}$. The partition function of the Ising model $Z_{G}(z, b)$ is defined as

$$
Z_{G}(z)=Z_{G}(z, b)=\sum_{U \subseteq V} z^{|U|} \cdot b^{|\delta(U)|}
$$

where $\delta(U)$ denotes the set of edges with one endpoint in $U$ and one endpoint in $U \backslash V$. In this paper, we fix $b>0$ and consider the partition function $Z_{G}(z)$ as a polynomial in $z$. The case $b<1$ is often referred to as the ferromagnetic case, while $b>1$ is referred to as the anti-ferromagnetic case.

In this paper, we will investigate the anti-ferromagnetic Ising model for the class of line graphs. The line graph of a graph $G$ is a graph $L(G)$ with vertices being the edges of $G$ and two edges being connected if they share a common vertex. In particular, in Section 4 we prove the following theorems.

Theorem 1. Let $G$ be a graph and let $b \geqslant 1$. Every root of $Z_{L(G)}(z, b)$ is real and negative.

We can also deduce some information on the location of the so-called Fisher zeros when we put a bound on the maximum degree. 
Theorem 2. For any $\Delta \geqslant 2$ and $0 \leqslant \alpha<\pi / 2$, there exists an open set $U_{\Delta, \alpha} \subseteq \mathbb{C}$ containing the interval $[1, \infty)$, such that if $G$ has maximum degree at most $\Delta$, then for $b \in U_{\Delta, \alpha}$ and $\lambda \in\{z \in \mathbb{C}|| \arg (z) \mid<\pi-2 \alpha\}$ we have

$$
Z_{L(G)}(\lambda, b) \neq 0 .
$$

In particular, $Z_{L(G)}(1, b) \neq 0$ for all $b \in U_{\Delta, \alpha}$.

A way to interpret the previous theorem is that there is no phase transition of the anti-ferromagnetic Ising-model on line graphs in the Fisher [14] sense. This phenomenon was already observed by Syôzi in [26] for the Kagomé-lattice, which is the line graph of the hexagonal lattice. Recently, a variant of the absence of phase transitions for line graphs was also proven by Dyer, Heinrich, Jerrum, and Müller [13]. In particular, they proved that for any choice of $b$ and $\lambda$, there exists a fully polynomial time randomized algorithm to approximate $Z_{L(G)}(\lambda, b)$. Using Theorem 2, one can show that with a uniform bound on the maximum degree, we can obtain a deterministic algorithm for the same task. Let us briefly explain. A successful approach for obtaining an approximation algorithm was proposed by Barvinok [4], based on truncating the Taylor series of the logarithm of partition functions over a connected zero-free domain. In [24], this method was improved so as to run in polynomial time on bounded degree graphs. By combining this approach (see also [22]) with the previous corollary and the existence of a zero-free disk around zero from [25, Remark 24], we obtain the following corollary.

Corollary 3. For $\Delta \geqslant 2$ and $0 \leqslant \alpha<\pi / 2$, let $U_{\Delta, \alpha}$ given by Theorem 2. Let $b \in U_{\Delta, \alpha}$ and $\xi \in\{z \in \mathbb{C}|| \arg (z) \mid<\pi-2 \alpha\}$. Then for any $\varepsilon>0$, there exists an algorithm that given an n-vertex graph $G$ of maximum degree at most $\Delta$, computes a multiplicative $\varepsilon$-approximation ${ }^{1}$ to $Z_{L(G)}(\xi, b)$ in time polynomial in $n / \varepsilon$.

\section{Edge cover polynomial}

The second polynomial we consider is the edge cover polynomial, which was introduced for graphs in [2]. We consider here the obvious extension to hypergraphs.

Let $\mathcal{H}=(V, E)$ be a hypergraph. A subset of edges $F \subseteq E$ is called an edge cover if each vertex of $\mathcal{H}$ is contained in at least one edge of $F$. We define the edge cover polynomial of $\mathcal{H}$ as

$$
\mathcal{E}(\mathcal{H}, z)=\sum_{F \subseteq E \text { edge cover }} z^{|F|} .
$$

In [11] it was proved that all the complex zeros of the edge cover polynomial of ordinary graphs are contained in the open disk of radius $\frac{(2+\sqrt{3})^{2}}{1+\sqrt{3}}$. Moreover, they showed that if the minimum degree is large enough, then the zeros are contained in the open disk of radius 4 . The authors conjectured that the actual bound will be 4 for all graphs, cf. [11, Conjecture 5.1].

Here we confirm this conjecture (see Section 3 for the proof):

\footnotetext{
${ }^{1} \mathrm{~A}$ multiplicative $\varepsilon$-approximation to a nonzero complex number $e^{a}$ is a number $e^{b}$ such that $|a-b| \leqslant \varepsilon$.
} 
Theorem 4. Let $\mathcal{H}$ be a hypergraph with largest edge of size $k$ without isolated vertices. Then

(i) $\mathcal{E}(\mathcal{H}, z) \neq 0$ if $|z|>2^{k}$,

(ii) if moreover $k=2$, then $\mathcal{E}(G, z) \neq 0$ if $|z| \geqslant 4$.

In fact we can prove a more refined result, which for graphs gives a zero-free region containing the positive real line. We state here only the result for graphs, see Section 3 for the extension to hypergraphs and its proof.

Theorem 5. Let $G$ be graph without isolated vertices. Then all roots of $\mathcal{E}(G, z)$ are contained in the set $\left\{-(1-\alpha)^{2}|| \alpha \mid \leqslant 1\right\}$.

See Figure 1 below for a picture of the set in Theorem 5 .

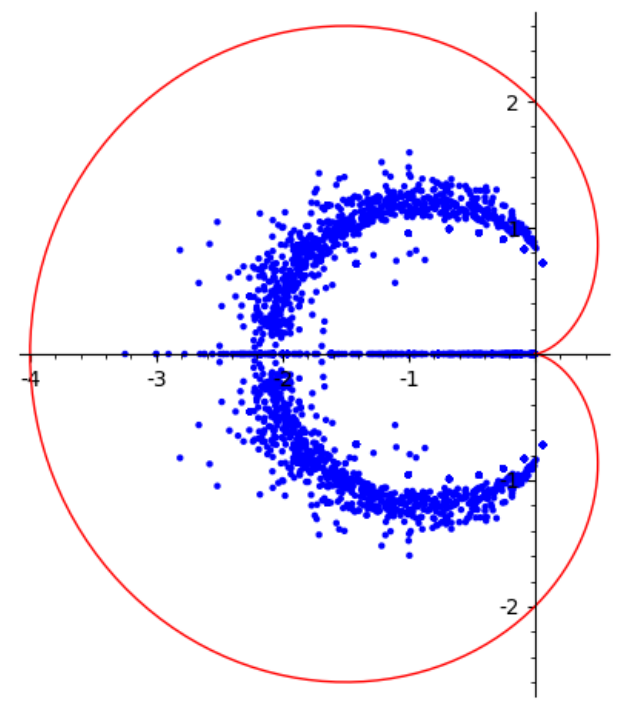

Figure 1: Roots of edge cover polynomial of some graphs on 10 vertices and the boundary of the set $\left\{-(1-\alpha)^{2}|| \alpha \mid \leqslant 1\right\}$.

Combining the theorem above with Barvinok's method [4] and the improvement from [24], we obtain a fully polynomial time approximation scheme for approximating the edge cover polynomial on the the complement of the set $\left\{-(1-\alpha)^{2}|| \alpha \mid \leqslant 1\right\}$ on bounded degree graphs. To do this one needs to interpolate from 'infinity'. Equivalently, one can interpolate the independence polynomial of the dual hypergraph (see below) from zero. We omit further details, but refer to [4, 24], noting that in [24] hypergraphs are not considered but the extension of the approach from [24] to hypergraphs is laid out in [22]. We note that on the real line this a gives a completely different algorithm than the one in $[20,21]$, which is based on decay of correlations. See also $[6,17]$ for randomized algorithms based on Markov chains. 


\section{Independent sets}

It is important to note that the notion of an edge cover of a hypergraph $\mathcal{H}$ is strongly related to independent sets of hypergraphs. We call a set of vertices $A \subseteq V$ an independent set, if no subset of $A$ forms an edge of $\mathcal{H}$ (in [5] this is called a weak independent set). In other words, a set $A$ is independent if and only if any edge of $\mathcal{H}$ contains at least one vertex from $V \backslash A$. Such a set is called a vertex cover. Let us denote the independence polynomial of $\mathcal{H}$ by

$$
I(\mathcal{H}, z)=\sum_{A \subseteq V \text { independent }} z^{|A|}
$$

A natural way to describe a vertex cover of a hypergraph $\mathcal{H}$ is to consider an edge cover in the dual hypergraph $\mathcal{H}^{T}$ with vertex set $E$ and edges $\{\{e \in E \mid v \in e\} \mid v \in V\}$. Then it is not hard to see that

$$
I(\mathcal{H}, z)=z^{|V|} \mathcal{E}\left(\mathcal{H}^{T}, 1 / z\right) .
$$

Thus we have the following corollary.

Corollary 6. Let $\mathcal{H}$ be a hypergraph of degree at most $\Delta$. If $|z|<2^{-\Delta}$, then

$$
I(\mathcal{H}, z) \neq 0 \text {. }
$$

\section{Domination polynomials}

As an additional application we obtain a bound on the roots of the total domination and domination polynomial that is independent of the number of vertices.

For a graph $G$ a set $S \subseteq V(G)$ is called a dominating set if for every $u \in V(G)$ we have either $u \in S$ or there exists a neighbor $v \in S$ of $u$. Let $d_{k}(G)$ denote the number of dominating sets of size $k$ in $G$. The domination polynomial is defined as $D(G, z)=\sum_{k} d_{k}(G) z^{k}$. See $[3,1,19]$ for further details.

Similarly, for a graph $G$ a set $S \subseteq V(G)$ is called a total dominating set if for every $u \in V(G)$ there exists a neighbor $v \in S$ of $u$. Let $d_{k}^{(t)}(G)$ denote the number of dominating sets of size $k$ in $G$. The total domination polynomial is defined as $D_{t}(G, z)=\sum_{k} d_{k}^{(t)}(G) z^{k}$. See $[9,12]$ for further details.

In [23] it is shown that $D(G, z)$ has all its complex zeros in a disk of radius $\sqrt[\delta+1]{2^{n}-1}$ around -1 . (Here $\delta$ denotes the minimum degree.) In [18] the authors showed a similar bound for $D_{t}(G, z)$, namely all the complex zeros are in the disk of radius $\sqrt[\delta]{2^{n}-1}$ around -1 . Observe that both bounds depend on the number of vertices of the graph.

For a graph $G$ and a vertex $v$ let $N_{G}(v)$ denote the set of neighbors of $v$, that is, $N_{G}(v)=\{u \in V \mid(u, v) \in E\}$. Let $N_{G}[v]=N_{G}(v) \cup\{v\}$, this is called the closed neighborhood of $v$. Let us define the hypergraph $\mathcal{D}_{G}\left(\operatorname{resp} . \mathcal{D}_{G, t}\right)$ on the vertex set $V(G)$ with edges $\left\{N_{G}[v] \mid v \in V(G)\right\}$ (resp. $\left\{N_{G}(v) \mid v \in V(G)\right\}$ ). Now observe that the (total) domination polynomial of $G$ is an edge cover polynomial of $\mathcal{D}_{G}$ (resp. $\mathcal{D}_{G, t}$ ), that is, $D(G, z)=\mathcal{E}\left(\mathcal{D}_{G}, z\right)$ and $D_{t}(G, z)=\mathcal{E}\left(\mathcal{D}_{G, t}, z\right)$. By specializing Theorem 4 we obtain that the roots of $D(G, z)$ (resp. $D_{t}(G, z)$ ) are contained in a disk of radius $2^{\Delta(G)+1}$ (resp. $2^{\Delta(G)}$ ) around 0 , assuming the graph $G$ has no isolated vertices. (Here $\Delta$ denotes the maximum degree.) 
Corollary 7. Let $G$ be a graph without isolated vertex and with maximum degree $\Delta$. Then the roots of the domination polynomial of $G$ are contained in the disk of radius $2^{\Delta(G)+1}$. The roots of the total domination polynomial of $G$ are contained in the disk of radius $2^{\Delta(G)}$.

\section{Organization}

In the next section we recall some notation and the main result of Wagner [27]. In Section 3 we prove our results for the edge cover polynomial, and in Section 4 we prove our results for the Ising model. In the final section we close with an open question and some remarks.

\section{Preliminaries}

Let us recall some notations and results of Wagner [27].

Let $\mathcal{A} \subset \mathbb{C}$. We say that a multivariate polynomial $p$ with variables $z_{1}, \ldots, z_{n}$ is $\mathcal{A}$-nonvanishing, if either $p$ is constant zero or $p\left(z_{1}, \ldots, z_{n}\right) \neq 0$ if all $z_{i} \in \mathcal{A}$.

Also we denote

- the sector $\mathcal{S}[\theta]=\{z \in \mathbb{C}|| \arg (z) \mid<\theta\}$ for some $0 \leqslant \theta<\pi$;

- the open interior of disk $\kappa \mathcal{D}=\{z \in \mathbb{C}|| z \mid<\kappa\}$ for some $0<\kappa$;

- the open exterior of a disk $\rho \mathcal{E}=\{z \in \mathbb{C}|| z \mid>\rho\}$ for some $0<\rho<\infty$.

In what follows we will use the degree sequence and the degree of a vertex. To emphasize the difference, we will use $\operatorname{deg}(\mathcal{H})$ for the degree sequence of a hypergraph $\mathcal{H}$, and $d_{\mathcal{H}}(v)$ for the degree of a vertex $v$. Let us fix a hypergraph $\mathcal{H}=(V, E)$. Then associate to each vertex $v$ of $\mathcal{H}$ a sequence of complex numbers $u^{(v)}=\left(u_{0}^{(v)}, \ldots, u_{d_{\mathcal{H}}(v)}^{(v)}\right)$ and to every edge $e$ associate a complex number $\lambda_{e}$. We define the (multivariate) subgraph counting polynomial of $\mathcal{H}$ with variables $x_{v}, v \in V$, as

$$
Z_{\mathcal{W}}(\mathcal{H}, \lambda, u ; x)=\sum_{F \subseteq E} \lambda^{F} u_{\operatorname{deg}(F)} x^{\operatorname{deg}(F)}
$$

where $\lambda^{F}=\prod_{e \in F} \lambda_{e}, u_{\operatorname{deg}(F)}=\prod_{v \in V} u_{d_{F}(v)}^{(v)}$ and $x^{\operatorname{deg}(F)}=\prod_{v \in V} x_{v}^{d_{F}(x)}$.

The strategy in [27] to obtain a zero-free region for $Z_{\mathcal{W}}(\mathcal{H}, \lambda, u ; x)$ is based on properties of two other polynomials. The first is the base polynomial of $\mathcal{H}$, defined as,

$$
\Omega(\mathcal{H}, \lambda, x)=\prod_{e \in E}\left(1+\lambda_{e} \prod_{v \in e} x_{v}\right)
$$

and the other is the key polynomial of a vertex $v$, defined as

$$
K^{(v)}(z)=\sum_{i=0}^{d_{\mathcal{H}}(v)}\left(\begin{array}{c}
d_{\mathcal{H}}(v) \\
i
\end{array}\right) u_{i}^{(v)} z^{i} .
$$

Now we are ready to state Wagner's theorem. 
Theorem 8 (Theorem 3.2 of [27]). Let $\mathcal{H}, u$, and $\lambda$ be defined as above.

(i) If $\Omega(\mathcal{H}, \lambda ; x)$ is $\mathcal{S}[\pi / 2]$-nonvanishing and for each vertex $v, K^{(v)}(z)$ is $\mathcal{S}[\pi-\alpha]$ nonvanishing, then $Z_{\mathcal{W}}(\mathcal{H}, \lambda, u ; x)$ is $\mathcal{S}[\pi / 2-\alpha]$-nonvanishing.

(ii) If $\Omega(\mathcal{H}, \lambda ; x)$ is $\kappa \mathcal{D}$-nonvanishing and for each vertex $v, K^{(v)}(z)$ is $\rho \mathcal{D}$-nonvanishing, then $Z_{\mathcal{W}}(\mathcal{H}, \lambda, u ; x)$ is $\kappa \rho \mathcal{D}$-nonvanishing.

(iii) If $\Omega(\mathcal{H}, \lambda ; x)$ is $\kappa \mathcal{E}$-nonvanishing and for each vertex $v, K^{(v)}(z)$ is $\rho \mathcal{E}$-nonvanishing and of degree $d_{\mathcal{H}}(v)$, then $Z_{\mathcal{W}}(\mathcal{H}, \lambda, u ; x)$ is $\kappa \rho \mathcal{E}$-nonvanishing.

We note that this result is only stated for graphs in [27], but the extension to hypergraphs that we present here is straightforward. For convenience of the reader we will provide a proof, closely following Wagner's proof for the graph case. Before we start, we would further like to make two remarks.

Remark 9. From the definition of $\Omega(\mathcal{H}, \lambda, x)$ it follows that if each hyperedge has size at least 2 , then $\Omega(\mathcal{H}, \lambda, x)$ is $\mathcal{S}[\pi / 2]$-nonvanishing if and only if each $\lambda_{e} \geqslant 0$ and the size of each hyperedge equals 2 . Therefore part (i) of the theorem only applies to ordinary graphs.

Remark 10. Another useful observation is the case when $\mathcal{H}$ is a $k$-uniform hypergraph. Using the substitution $x_{v}=z^{1 / k}$ for each vertex $v$ of $\mathcal{H}$, the polynomial

$$
Z_{\mathcal{W}}(\mathcal{H}, \lambda, u ; x)=\sum_{F \subseteq E} \lambda^{F} u_{\operatorname{deg}(F)} z^{|F|}
$$

is a one variable polynomial, since in a $k$-uniform hypergraph $\sum_{v \in V} d_{F}(v)=k|F|$.

To prove Theorem 8 we will need the following lemma.

Lemma 11 (Schur-Szegó, Proposition 2.4(b) and (c) of [27]). Let $P(z)=\sum_{j} c_{j} z^{j}$ and $K(z)=\sum_{j=0}^{d}\left(\begin{array}{l}d \\ j\end{array}\right) u_{j} z^{j}$ be polynomials in one complex variable, with $\operatorname{deg} P \leqslant d$. The SchurSzegó composition of polynomials $P(z)$ and $K(z)$ is the polynomial $Q(z)=\sum_{j=0}^{d} u_{j} c_{j} z^{j}$. For any $\kappa>0$ and $\rho>0$, if $P(z)$ is $\rho \mathcal{D}$-nonvanishing and $K(z)$ is $\kappa \mathcal{D}$-nonvanishing, then $Q(z)$ is $\kappa \rho \mathcal{D}$-nonvanishing. Similarly, if $P(z)$ is $\rho \mathcal{E}$-nonvanishing and $K(z)$ is $\kappa \mathcal{E}$ nonvanishing and $\operatorname{deg}(K)=d$, then $Q(z)$ is $\kappa \rho \mathcal{E}$-nonvanishing.

Proof of Theorem 8. By Remark 9 above, part (i) is covered by Theorem 3.2 of [27]. We therefore focus on the case that $\Omega(\mathcal{H}, \lambda ; x)$ is $\kappa \mathcal{D}$-nonvanishing and each key polynomial $K^{(v)}(z)$ is $\rho \mathcal{D}$-nonvanishing. The proof for the case where $\mathcal{D}$ is replaced by $\mathcal{E}$ follows along exactly the same lines.

We identify the vertex set $V$ with $\{1, \ldots, n\}$. We define a sequence of polynomials, $F_{0}(x), F_{1}(x), \ldots, F_{n}(x)$ as follows. We set $F_{0}(x):=\Omega(\mathcal{H}, \lambda, x)$. For all $1 \leqslant i \leqslant n$ we let $F_{i}(x)$ to be obtained as the Schur-Szegó composition of $F_{i-1}(x)$ and the $i$ th key polynomial in the variable $x_{i}, K_{i}\left(x_{i}\right)$ (the remaining variables being absorbed in the coefficients). By induction one has

$$
F_{i}(x)=\sum_{F \subseteq E} \lambda^{F}\left(\prod_{j=1}^{i} u_{d_{F}(j)}^{(j)}\right) x^{\operatorname{deg}(F)},
$$


implying that $F_{n}(x)=Z_{\mathcal{W}}((\mathcal{H}, \lambda, u ; x))$.

We next show by induction that if $\xi_{1}, \ldots, \xi_{n}$ are such that

$$
\xi_{j} \in \kappa \rho \mathcal{D} \text { if } j<i \text { and } \xi_{j} \in \rho \mathcal{D} \text { if } j>i,
$$

then $F_{i-1}\left(\xi_{1}, \ldots, \xi_{j-1}, x_{i}, \xi_{i+1}, \ldots, \xi_{n}\right)$ is $\rho \mathcal{D}$-nonvanishing. The base case $i=1$ follows from the assumption. By Lemma 11 we immediately obtain the induction step. To see that $F_{n}(x)$ is $\kappa \rho \mathcal{D}$-nonvanishing, we apply Lemma 11 once more to $F_{n-1}\left(\xi_{1}, \ldots, \xi_{n-1}, x_{n}\right)$ and $K_{n}\left(x_{n}\right)$, for any choice of $\xi_{1}, \ldots, \xi_{n-1} \in \kappa \rho \mathcal{D}$, which is $\kappa \mathcal{D}$-nonvanishing by the above, to obtain the desired result.

Our main goal will be to express the partition function of the Ising-model of a line graph and the edge cover polynomial as a subgraph counting polynomial. We start with the edge cover polynomial, as this is easiest one.

\section{$3 \quad$ Edge cover polynomial}

We start by giving two proofs of Theorem 4(i), by expressing the edge cover polynomial as a subgraph counting polynomial in two different ways. After this we prove Theorem 5 and we conclude with proving that for graphs -4 cannot be a root of the edge cover polynomial thereby concluding the proof of Theorem 4(ii).

\subsection{First proof of Theorem 4(i)}

Lemma 12. For a hypergraph $\mathcal{H}$, the edge cover polynomial at $\xi$ can be expressed as

$$
\mathcal{E}(\mathcal{H}, \xi)=Z_{\mathcal{W}}(\mathcal{H}, \xi,(0,1, \ldots, 1) ; 1) .
$$

Proof. We simply have to check the definition of the subgraph counting polynomial, that is,

$$
Z_{\mathcal{W}}(\mathcal{H}, \xi,(0,1, \ldots, 1) ; 1)=\sum_{F \subseteq E} \xi^{|F|} \prod_{v \in V(\mathcal{H})} \mathbf{1}_{d_{F}(v)>0}=\mathcal{E}(\mathcal{H}, \xi)
$$

To apply Wagner's theorem, we have to investigate the location of zeros of the key polynomials. Let $L_{d}(z)=(1+z)^{d}$ and $K_{d}(z)=(1+z)^{d}-1$.

Lemma 13. For any $d \geqslant 0$, the polynomials

$$
L_{d}(z)=(1+z)^{d} \quad \text { and } \quad K_{d}(z)=\sum_{i=1}^{d}\left(\begin{array}{l}
d \\
i
\end{array}\right) z^{i}=(1+z)^{d}-1
$$

are $2 \mathcal{E}$-nonvanishing.

Proof. The statement is trivial for $L_{d}(z)$. On the other hand, the roots of $K_{d}(z)$ are translations of the $d$-th root of unity by 1 . Since the $d$-th roots of unity form vertices of a regular $d$-gon, therefore by simple geometric argument, we obtain the desired statement. 
Let us fix a value $\xi \in \mathbb{C}$, such that $\kappa=|\xi|>2^{k}>1$.

Lemma 14. The base polynomial,

$$
\prod_{e \in E}\left(1+\xi \prod_{v \in e} x_{v}\right)
$$

is $\kappa^{-1 / k} \mathcal{E}$-nonvanishing.

Proof. This is clear since if each $x_{v}$ has absolute value at least $\kappa^{-1 / k}$, then

$$
\left|\xi \prod_{v \in e} x_{v}\right|>\kappa \prod_{v \in e} \kappa^{-1 / k}=\kappa^{1-|e| / k} \geqslant 1 .
$$

Now we are ready to prove Theorem $4(\mathrm{i})$. We would like to show that $\mathcal{E}(\mathcal{H}, \xi) \neq 0$. Consider the polynomial $Z_{\mathcal{W}}(\mathcal{H}, \xi,(0,1, \ldots, 1), z)$. We will use Theorem 8 to show that the subgraph counting polynomial is not zero at $z=1$. Indeed, as the key polynomials are $2 \mathcal{E}$-nonvanishing, we get from Theorem 8 that $Z_{\mathcal{W}}(\mathcal{H}, \xi,(0,1, \ldots, 1), z)$ is $2\left(\kappa^{-1 / k}\right) \mathcal{E}$ nonvanishing, that is $(1-\varepsilon) \mathcal{E}$-nonvanishing for some $\varepsilon>0$. As we are interested in the value of this polynomial at $z=1$, and since $1 \in(1-\varepsilon) \mathcal{E}$, we therefore obtain,

$$
0 \neq Z_{\mathcal{W}}(\mathcal{H}, \xi,(0,1, \ldots, 1), 1)=\mathcal{E}(\mathcal{H}, \xi)
$$

as desired.

\subsection{Second proof of Theorem 4(i)}

It will be convenient for us to define for a hypergraph $\mathcal{H}=(V, E)$ of largest edge size $k$, its uniformization, $\widehat{\mathcal{H}}$, by adding new extra vertices to edges, in a way that we obtain a $k$-uniform hypergraph with same number of edges. Let the set of new vertices be denoted by $S$ and the set of edges of $\widehat{\mathcal{H}}$ by $\widehat{E}$.

Lemma 15. For a hypergraph $\mathcal{H}$ the edge cover polynomial can be expressed as

$$
\mathcal{E}(\mathcal{H}, z)=Z_{\mathcal{W}}\left(\widehat{\mathcal{H}}, 1, u ; z^{1 / k}\right)
$$

where $u^{(v)}=(0,1,1, \ldots, 1)$ if $v \notin S$ and $u^{(v)}=(1, \ldots, 1)$ otherwise.

Proof. Observe that a subset of edges $E$ in $\mathcal{H}$ is an edge cover if and only if the corresponding edges in $\widehat{\mathcal{H}}$ covers $V(\mathcal{H}) \backslash S$ as well. Thus the edge covering polynomial of $\mathcal{H}$ and the "relaxed edge cover polynomial" of $\widehat{\mathcal{H}}$ are the same. The lemma is now an immediate corollary of the definition of the edge cover polynomial and Remark 10 in the previous section. 
In order to apply Wagner's theorem, we have to investigate the location of zeros of the key polynomials $L_{d}(z)=(1+z)^{d}$ and $K_{d}(z)=\sum_{i=1}^{d}\left(\begin{array}{c}d \\ i\end{array}\right) z^{i}=(1+z)^{d}-1$. We already proved the relevant properties of these polynomials in Lemma 13. Using this, we obtain by Theorem 8 , that

$$
Z_{\mathcal{W}}(\widehat{\mathcal{H}}, 1, u ; z)=\sum_{F \subseteq \widehat{E} \text { covers } V(\mathcal{H}) \backslash S} z^{\sum_{v \in \widehat{V}} d_{F}(v)}=\sum_{F \subseteq \widehat{E} \text { covers } V(\mathcal{H}) \backslash S} z^{k|F|}
$$

is $2 \mathcal{E}$-nonvanishing. By substituting $z \mapsto z^{1 / k}$, we obtain a polynomial that is $2^{k} \mathcal{E}$ nonvanishing, as desired.

\subsection{The Cardioid-like region}

In this section, we will strengthen Theorem 4 . We will not use Theorem 8 , but a similar technique to find a zero-free region for the subgraph-counting function.

The following lemma will play the role of Asano-contraction in the main proof. This lemma is a slight modification of [15, Lemma 7].

Lemma 16. Let $p(z)=\sum_{k=0}^{d} a_{k} z^{k}$ such that $a_{d} \neq 0$. Assume that $p(z) \neq 0$ if $z \notin K$ for some $K \subseteq \mathbb{C}$ closed set. Then

$$
q(z)=a_{d} z+a_{0}
$$

has its (unique) root contained in $(-1)^{d+1} K^{d}$.

Proof. Let $p(z)=a_{d}\left(z+\xi_{1}\right) \ldots\left(z+\xi_{d}\right)$, where $\xi_{i} \in-K$. If we denote the root of $q(z)$ by $z_{0}$, then

$$
z_{0}=-\frac{a_{0}}{a_{d}}=-\frac{a_{d} \xi_{1} \ldots \xi_{d}}{a_{d}} \in(-1)^{d+1} K^{d}
$$

We will call the previous polynomial transformation the Asano-contraction of $p(z)$ over the variable $z$ of degree $d$.

The idea of the proof is that we use Asano-contraction iteratively for a rightly chosen multivariate polynomial. The issue that could occur is that the resulting polynomial does not have the correct degree so that we cannot apply Asano-contraction again. To rule out this case, we have to relax the definition of the edge cover polynomial by saying there are vertices where we can use any number of edges similarly to the subgraph counting polynomial of the previous subsection.

Definition 17. Let $\mathcal{H}$ be a hypergraph with $E(\mathcal{H})=\left\{e_{1}, \ldots, e_{m}\right\}$ edges, and let $S$ be a subset of $V(\mathcal{H})$. Then we define

- the base-polynomial

$$
\Omega_{\mathcal{H}, S}\left(z_{e_{1}}, \ldots, z_{e_{m}}\right)=\prod_{v \in S}\left(\prod_{e: v \in e}\left(1+z_{e}\right)\right) \prod_{v \notin S}\left(\prod_{e: v \in e}\left(1+z_{e}\right)-1\right)
$$


- for $0 \leqslant k \leqslant m$ the intermediate polynomials as

$$
P_{\mathcal{H}, S, 0}(\mathbf{z})=\Omega_{\mathcal{H}, S}(\mathbf{z}),
$$

and $P_{\mathcal{H}, S, k}(\mathbf{z})$ as the Asano-contraction of $P_{\mathcal{H}, S, k-1}(\mathbf{z})$ over the variable $z_{e_{k}}$ of degree $\left|e_{k}\right|$

- the edge-cover polynomial of $\mathcal{H}$ relaxed over $S$ as

$$
\mathcal{E}(\mathcal{H}, S, z)=P_{\mathcal{H}, S, m}(z, \ldots, z)
$$

- $B=\{\alpha \in \mathbb{C}|| \alpha+1 \mid>1\}$, and $T_{k}=\mathbb{C} \backslash\left\{(-1)^{k+1} \prod_{i=1}^{k} \alpha_{i} \mid \alpha_{1}, \ldots, \alpha_{k} \notin B\right\}$, in particular, $T_{1}=B$

- Notation: $T_{\mathcal{H}, k}=T_{\left|e_{1}\right|} \times \cdots \times T_{\left|e_{k}\right|} \subseteq \mathbb{C}^{k}$.

Remark 18. It is important to understand the meaning of $P_{\mathcal{H}, S, m}\left(z_{e_{1}}, \ldots, z_{e_{m}}\right)$. Note that it is a multilinear polynomial since after an application of the Asano contraction to the variable $z_{e}$ the resulting polynomial will be linear in this variable. So this polynomial can be written as

$$
\sum_{F \subseteq E(\mathcal{H})} a_{F} \prod_{e \in F} z_{e}
$$

Now let us understand the coefficient $a_{F}$. This means that whenever $e \in F$ we chose the degree $|e|$ term from the previous multivariate polynomial, and whenever $e \notin F$ we chose the constant term. Now observe that if $v \notin S$, then there is no constant term in $\prod_{v \in e}\left(1+z_{e}\right)-1$. This means that we should choose at least one edge covering $v$ into $F$, otherwise $a_{F}=0$. For $v \in S$ there is no such requirement since there is a constant term in $\prod_{v \in e}\left(1+z_{e}\right)$. So $a_{F}=1$ if the elements of $F$ cover every vertex not in $S$. They may cover some vertices from $S$, but they do not need to. So the meaning of

$$
\mathcal{E}(\mathcal{H}, S, z)=P_{\mathcal{H}, S, m}(z, \ldots, z)
$$

is that it counts the edge sets $F$ with weight $z^{|F|}$ if it covers all vertices not in $S$. Thus $\mathcal{E}(\mathcal{H}, \varnothing, z)$ is the classical edge cover polynomial of the hypergraph $\mathcal{H}$.

Theorem 19. Let $\mathcal{H}=(V, E)$ be a hypergraph. For any set $S \subseteq V$ containing all isolated vertices of $\mathcal{H}$, and $k \geqslant 0$, the polynomial

$$
P_{\mathcal{H}, S, k}(\mathbf{z})
$$

is non-zero and $T_{\mathcal{H}, k} \times B^{m-k}$-nonvanishing.

Proof. We will prove the statement by induction on $k$. If $k=0$, then the statement is trivial for any $S \subseteq V(G)$, since for any vertex the polynomials

$$
\prod_{v \in e}\left(1+z_{e}\right) \text { and } \prod_{v \in e}\left(1+z_{e}\right)-1
$$


are $B^{m}$-stable.

Let us assume that $k \geqslant 1$. Then, by induction, we have $P_{\mathcal{H}, S, k-1}(\mathbf{z})$ is $T_{\mathcal{H}, k-1} \times B^{m-k+1}$ nonvanishing non-zero polynomial. Fix $\left(\xi_{1}, \ldots, \xi_{k-1}\right) \in T_{\mathcal{H}, k-1}$, and $\tau_{k+1}, \ldots, \tau_{m} \in B$. Then,

$$
p(z)=P_{\mathcal{H}, S, k-1}\left(\xi_{1}, \ldots, \xi_{k-1}, z, \tau_{k+1}, \ldots, \tau_{m}\right)
$$

is a non-zero $B$-stable polynomial or the zero-polynomial.

We claim that $p(z)$ is a polynomial of degree $\left|e_{k}\right|$. This is true, since the coefficient of $z^{\left|e_{k}\right|}$ in $p(z)$ is exactly

$$
P_{\mathcal{H}-e_{k}, S \cup e_{k}, k-1}\left(\xi_{1}, \ldots, \xi_{k-1}, \tau_{k+1}, \ldots, \tau_{m}\right),
$$

which is not the zero polynomial by induction. (Since new additional isolated vertices of $\mathcal{H}-e_{k}$ are in $e_{k}$.)

If we denote

$$
q(z)=P_{\mathcal{H}, S, k}\left(\xi_{1}, \ldots, \xi_{k-1}, z, \tau_{k+1}, \ldots, \tau_{m}\right),
$$

then $q(z)$ is exactly the Asano-contraction of $p(z)$ over $z$ of degree $\left|e_{k}\right|$. By Lemma 16 we have that $q(z) \neq 0$ if $z \in T_{\left|e_{k}\right|}$.

Thus, we proved that for any choice of $\left(\xi_{1}, \ldots, \xi_{k-1}, \xi_{k}\right) \in T_{\mathcal{H}, k}$ and $\tau_{k+1}, \ldots, \tau_{m} \in B$, the polynomial

$$
P_{\mathcal{H}, S, k}\left(\xi_{1}, \ldots, x_{k}, \tau_{k+1}, \ldots, \tau_{m}\right) \neq 0,
$$

as desired.

Theorem 5 follows directly from this result, taking $S=\varnothing$ and realizing that the set $T_{2}$ is equal to the complement of the set $\left\{-(1-\alpha)^{2}|| \alpha \mid \leqslant 1\right\}$, see the next lemma.

Lemma 20. The set $T_{2} \subseteq \mathbb{C}$ is exactly $\mathbb{C} \backslash\left\{-(1-\alpha)^{2}|| \alpha \mid \leqslant 1\right\}$.

In the forthcoming proof $\measuredangle B A C$ means the angle at $A$ determined by the lines $B A$ and $A C$.

Proof. The statement is equivalent with the the following statement. Let $R=\{z||(-1)-$ $z \mid \leqslant 1\}$, then the sets $R_{1}=\left\{z^{2} \mid z \in R\right\}$ and $R_{2}=\left\{z_{1} z_{2} \mid z_{1}, z_{2} \in R\right\}$ are the same. Clearly, $R_{1} \subseteq R_{2}$ so we only need to prove the opposite containment. Note that both $R_{1}$ and $R_{2}$ are star convex from the point 0 , so it is enough to prove that if $w \in R_{2}$, then there exists a $w^{\prime}$ such that $\arg \left(w^{\prime}\right)=\arg (w)$ and $\left|w^{\prime}\right| \geqslant|w|$. So we assume that $z_{1}, z_{2} \in R$ are on the boundary of $R$, and $w=z_{1} z_{2}$. We show that if we choose $z^{\prime}$ to be the intersection of the boundary of $R$ with the angle bisector of the angle determined by $z_{1}, 0, z_{2}$, then $w^{\prime}=z^{\prime 2}$ satisfies the above conditions.

To see this we need the following geometric fact. Let $A B C$ be a triangle and let $D$ be the intersection of the circumscribed circle of $A B C$ and the angle bisector of $\angle B A C$. Let $E$ be the intersection of the angle bisector of $\angle B A C$ and the side $B C$. Then $|A B| \cdot|A C|=$ $|A D| \cdot|A E|$. This is because the triangles $A B D$ and $A E C$ are similar: $\measuredangle B A D=\measuredangle E A C$ since $A D$ is an angle bisector, and $\measuredangle A D B=\measuredangle A C E$ by the inscribed angle theorem (see Figure 2 for a picture describing this). Hence $|A B| \cdot|A C|=|A D| \cdot|A E| \leqslant|A D|^{2}$.

Applying this to $A=0, B=z_{1}, C=z_{2}, D=z^{\prime}$ we get the claim that for $w=z_{1} z_{2}$ and $w^{\prime}=z^{\prime 2}$ we have $\arg \left(w^{\prime}\right)=\arg (w)$ and $\left|w^{\prime}\right| \geqslant|w|$. 


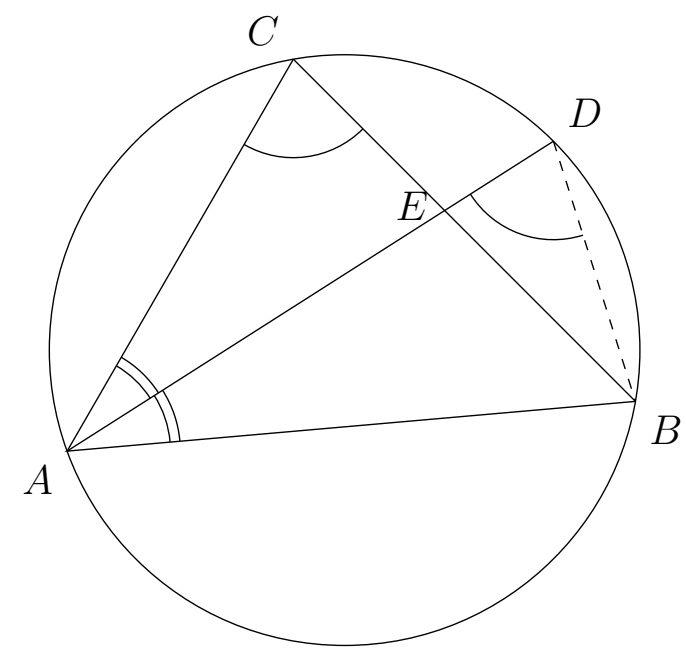

Figure 2: Proof of the geometric fact from Lemma 20.

\subsection{The evaluation at $z_{0}=-4$}

The main goal of this subsection is to finish the proof of Theorem 4(ii) and thereby confirming Conjecture 5.1 of [11]. By Theorem 5 a root of the edge cover polynomial can have absolute value 4 only if it is equal to -4 . As we will show, this case cannot occur.

We need the following lemma.

Lemma 21. Let $G=(V, E)$ be a multigraph on $m$ edges and let $S \subset V$ be a set of vertices containing all isolated vertices of $G$. If $\mathcal{E}(G, S,-4)=0$ and $e=(u, v) \in E$, then either $\mathcal{E}(G-e, S \cup\{u, v\},-4)=0$ or $e$ is not a loop and $\mathcal{E}(G / e, S,-4)=0$.

Proof. Suppose for contradiction that $\mathcal{E}(G-e, S \cup\{u, v\},-4) \neq 0$ and if $e$ is not a loop, then $\mathcal{E}(G / e, S,-4) \neq 0$. Assume that the edges are ordered in a way such that $e_{m}=e$. To proceed, we have to discuss a few cases depending the size of $e$, and on the number of endpoints of $e=(u, v)$ being in $S$.

If $u=v$, that is, $e$ is a loop, then the polynomial

$$
P_{G, S, m}(-4, \ldots,-4, z)
$$

is a linear $T_{1}=B$-nonvanishing polynomial that is zero at -4 . This could only happen, if this is the zero polynomial, in which case the 'main coefficient' $\mathcal{E}(G-e, S \cup\{u\},-4)=0$, a contradiction.

For the remainder we may assume that $u \neq v$. If $u, v \in S$, then we have

$$
0=\mathcal{E}(G, S,-4)=(-4+1) \cdot \mathcal{E}(G-e, S,-4) .
$$

Thus $\mathcal{E}(G-e, S \cup\{u, v\},-4)=0$, a contradiction.

So we may assume that not both $u$ and $v$ are contained in $S$. The polynomial

$$
q(z)=P_{G, S, m}(-4, \ldots,-4, z),
$$


is a non-zero linear polynomial with a zero at -4 . Indeed, since the main coefficient is $\mathcal{E}(G-e, S \cup\{u, v\},-4) \neq 0$. Next, consider the polynomial

$$
p(z)=P_{G, S, m-1}(-4, \ldots,-4, z) .
$$

This is a polynomial of degree 2, which is $B$-nonvanishing. Since $q(z)$ is the Asanocontraction of $p(z)$, the two zeros $\xi_{1}, \xi_{2}$ of $p(z)$ satisfy $\xi_{1} \xi_{2}=4$. On the other hand, we know that $p(z)$ is a $B$-nonvanishing polynomial, therefore, $\xi_{1}=\xi_{2}=-2$. In particular, the constant term and the linear term of $p(z)$ are equal. We obtain that the constant term is equal to $\mathcal{E}(G-e, S,-4)$ and the linear term is equal to $\mathcal{E}(G-e, S \cup\{u\},-4)+$ $\mathcal{E}(G-e, S \cup\{v\},-4)$. Therefore we obtain in case $u, v \notin S$, that $\mathcal{E}(G-e, S,-4)$ equals

$$
\begin{aligned}
& \mathcal{E}(G-e, S \cup\{u\},-4)+\mathcal{E}(G-e, S \cup\{v\},-4) \\
= & (\mathcal{E}(G-e, S,-4)+\mathcal{E}(G-u, S,-4))+(\mathcal{E}(G-e, S,-4)+\mathcal{E}(G-v, S,-4)) .
\end{aligned}
$$

From which it follows that

$$
0=\mathcal{E}(G-e, S,-4)+\mathcal{E}(G-u, S,-4)+\mathcal{E}(G-v, S,-4)=\mathcal{E}(G / e, S,-4),
$$

as desired. Otherwise, we may assume by symmetry, that $u \in S$ and $v \notin S$, in which case we obtain that $\mathcal{E}(G-e, S,-4)$ equals

$$
\begin{aligned}
& \mathcal{E}(G-e, S \cup\{u\},-4)+\mathcal{E}(G-e, S \cup\{v\},-4) \\
= & \mathcal{E}(G-e, S,-4)+\mathcal{E}(G-e, S \cup\{u, v\},-4),
\end{aligned}
$$

implying that $\mathcal{E}(G-e, S \cup\{u, v\},-4)=0$, a contradiction. This finishes the proof.

As an immediate corollary we obtain that -4 cannot be a root of the edge cover polynomial.

Corollary 22. Let $G=(V, E)$ be a multigraph and let $S \subseteq V$ be a set containing all isolated vertices of $G$. Then

$$
\mathcal{E}(G, S,-4) \neq 0
$$

Proof. For the sake of contradiction, assume that there exists such an example. Let $G$ be a counterexample with the minimum number of edges. If $G$ has no edge, then $S=V$, thus, $\mathcal{E}(G, S, z)=1$. Therefore, we may assume that $G$ has an edge. Let $e \in E(G)$. Then, by the previous lemma, either $E(G-e, S \cup\{u, v\},-4)=0$ or $E(G / e, S,-4)=0$. But in each case, the number of edges is strictly less than the number of edges of $G$ that leads us to a contradiction.

\section{The antiferromagnetic Ising-model on line graphs}

We begin by expressing the partition function of the Ising model on a line graph in terms of the underlying graph. 
Lemma 23. For a line graph $L=L(G)$, the partition function of the Ising model has the following form

$$
Z_{L(G)}\left(z^{2}\right)=b^{|E(L)|} \sum_{F \subseteq E} \prod_{v \in V} b^{-\left({ }^{d_{G}(v)-d_{F}(v)}\right)-\left({ }^{d_{F}(v)}\right)} z^{d_{F}(v)}
$$

Proof. Since the vertex set of $L(G)$ coincides with the edges of $G$, therefore

$$
Z_{L(G)}\left(z^{2}, b\right)=\sum_{U \subseteq V(L(G))} z^{2|U|} \cdot b^{\left|\delta_{L(G)}(U)\right|}=\sum_{F \subseteq E(G)} z^{2|F|} \cdot b^{\left|\delta_{L(G)}(F)\right|} .
$$

To express $\delta_{L(G)}(F)$, observe that $L(G)$ can be covered by edge disjoint cliques $Q_{v}$, where each clique corresponds those edges of $G$ containing the vertex $v$. Therefore, to count the number of edges in $\delta_{L(G)}(F)$ we can take the disjoint union

$$
\delta_{L(G)}(F)=\bigcup_{v \in V(G)} E\left(Q_{v}\right) \cap \delta_{L(G)}(F) .
$$

Let us assume that $Q_{v}$ is a clique of size $d$ and $\left|F \cap Q_{v}\right|=f$, i.e. $\operatorname{deg}_{G}(v)=d$ and $\operatorname{deg}_{F}(v)=f$. Then clearly,

$$
\left|E\left(Q_{v}\right) \cap \delta_{L(G)}(F)\right|=f(d-f)=\left(\begin{array}{l}
d \\
2
\end{array}\right)-\left(\begin{array}{l}
f \\
2
\end{array}\right)-\left(\begin{array}{c}
d-f \\
2
\end{array}\right) .
$$

Thus,

$$
\left|\delta_{L(G)}(F)\right|=\sum_{v \in V(G)}\left[\left(\begin{array}{c}
d_{G}(v) \\
2
\end{array}\right)-\left(\begin{array}{c}
d_{F}(v) \\
2
\end{array}\right)-\left(\begin{array}{c}
d_{G}(v)-d_{F}(v) \\
2
\end{array}\right)\right]
$$

and

$$
\begin{aligned}
Z_{L(G)}\left(z^{2}\right) & =\sum_{F \subseteq E(G)} b^{\sum_{v \in V(G)}\left({ }_{G_{2}}(v)\right)-\left({ }^{d_{F}(v)}\right)-\left({ }_{d_{G}(v)-d_{F}(v)}\right)} z^{\sum_{v} \operatorname{deg}_{F}(v)} \\
& =b^{|E(L(G))|} \sum_{F \subseteq E(G)} \prod_{v \in V} b^{-\left({ }^{\left(d_{G}(v)-d_{F}(v)\right.}\right)-\left({ }^{d_{F}(v)}\right)} z^{d_{F}(v)}
\end{aligned}
$$

We now observe that

$$
Z_{L(G)}(z)=b^{|E(L(G))|} Z_{\mathcal{W}}\left(G, 1,\left(b^{\left.\left.-\left(\begin{array}{c}
d_{G}(v)-i \\
2
\end{array}\right)-\left(\begin{array}{c}
i \\
2
\end{array}\right)\right)_{i=0}^{d_{G}(v)} ; z^{1 / 2}\right) .}\right.\right.
$$

The relevant base polynomial is given by for a graph $G=(V, E)$,

$$
\Omega(G, 1, x)=\prod_{e \in E}\left(1+\prod_{v \in e} x_{v}\right)
$$

and is clearly $\mathcal{S}[\pi / 2]$-nonvanishing (and $\mathcal{D}$-nonvanishing). Therefore, by Theorem 8 , to prove Theorem 1, it is enough to show that the key polynomials have only real roots, as we will show in the next lemma. 
For the remainder of this section, let us for fixed $b>1$, denote the relevant key polynomials by

$$
K_{d}(z)=\sum_{i=0}^{d}\left(\begin{array}{l}
d \\
i
\end{array}\right) b^{-\left(\begin{array}{c}
d-i \\
2
\end{array}\right)-\left(\begin{array}{c}
i \\
2
\end{array}\right) z^{i}}
$$

Lemma 24. For any $d \geqslant 0$ we have

$$
K_{d+1}(z)=b^{-d} K_{d}(b z)+z K_{d}\left(b^{-1} z\right) .
$$

Proof. By definition,

$$
\begin{aligned}
K_{d+1}(z) & =\sum_{i=0}^{d+1}\left(\begin{array}{c}
d+1 \\
i
\end{array}\right) b^{-\left(\begin{array}{c}
d+1-i \\
2
\end{array}\right)-\left(\begin{array}{c}
i \\
2
\end{array}\right) z^{i}} \\
& =\sum_{i=0}^{d+1}\left(\left(\begin{array}{l}
d \\
i
\end{array}\right)+\left(\begin{array}{c}
d \\
i-1
\end{array}\right)\right) b^{-\left(\begin{array}{c}
d+1-i \\
2
\end{array}\right)-\left(\begin{array}{c}
i \\
2
\end{array}\right)} z^{i} \\
& =\sum_{i=0}^{d}\left(\begin{array}{c}
d \\
i
\end{array}\right) b^{-\left(\begin{array}{c}
d+1-i \\
2
\end{array}\right)-\left(\begin{array}{c}
i \\
2
\end{array}\right)} z^{i}+\sum_{i=0}^{d}\left(\begin{array}{c}
d \\
i
\end{array}\right) b^{-\left(\begin{array}{c}
d-i \\
2
\end{array}\right)-\left(\begin{array}{c}
i+1 \\
2
\end{array}\right)} z^{i+1} \\
& =\sum_{i=0}^{d}\left(\begin{array}{c}
d \\
i
\end{array}\right) b^{-\left(\begin{array}{c}
d-i \\
2
\end{array}\right)-\left(\begin{array}{c}
i \\
2
\end{array}\right)} b^{-\left(\begin{array}{c}
d-i \\
1
\end{array}\right)} z^{i}+z \sum_{i=0}^{d}\left(\begin{array}{c}
d \\
i
\end{array}\right) b^{-\left(\begin{array}{c}
d-i \\
2
\end{array}\right)-\left(\begin{array}{c}
i \\
2
\end{array}\right)} b^{-\left(\begin{array}{c}
i \\
1
\end{array}\right)} z^{i} \\
& =b^{-d} K_{d}(b z)+z K_{d}\left(b^{-1} z\right) .
\end{aligned}
$$

Lemma 25. For any real number $b>1$ and integer $d \geqslant 1$ the key polynomial

$$
K_{d}(z)=\sum_{i}\left(\begin{array}{l}
d \\
i
\end{array}\right) b^{-\left(\begin{array}{c}
d-i \\
2
\end{array}\right)-\left(\begin{array}{c}
i \\
2
\end{array}\right)} z^{i}
$$

has simple negative real roots, i.e., it is $\mathcal{S}[\pi]$-nonvanishing. Moreover, the largest root $z_{0}^{(d)}<0$ of $K_{d}(z)$ has absolute value at most $\left|z_{0}^{(d)}\right|<b^{-d}$.

Proof. To prove the statement, we will show the following stronger statement: For any $d \geqslant 1$ the polynomial $K_{d}(z)$ has distinct zeros $0>z_{1}^{(d)}>\cdots>z_{d}^{(d)}$ and for any $1 \leqslant i \leqslant$ $d-1$

$$
\frac{z_{i+1}^{(d)}}{z_{i}^{(d)}}>b^{2} .
$$

We will prove this statement by induction on $d$ using the identity of Lemma 24, namely, $K_{d+1}(z)=b^{-d} K_{d}(b z)+z K_{d}\left(b^{-1} z\right)$. If $d=1$, then the above statement on the zeros trivially holds. Assume that the statement is true for some $d \geqslant 1$. Let $P_{1}(z)=$ $K_{d}(b z)$ and $P_{2}(z)=z K_{d}\left(b^{-1} z\right)$ and let $a_{1}>a_{2}>\cdots>a_{d}$ be the zeros of $P_{1}(z)$ and let $0=b_{1}>b_{2}>\cdots>b_{d+1}$ be the zeros of $P_{2}(z)$. We refer to Figure 3 for a schematic figure displaying the ideas of the proof. 


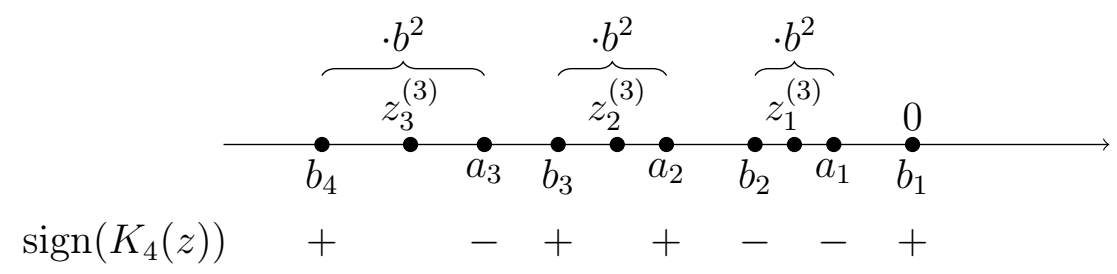

Figure 3: A schematic figure of the location of the zeros of $K_{3}(z), P_{1}(z)$ and $P_{2}(z)$.

We claim that $a_{i}>b_{i+1}$ and $b_{i}>a_{i}$ for any $0 \leqslant i \leqslant d$. This is true indeed, since

$$
\frac{a_{i}}{b_{i+1}}=\frac{b^{-1} z_{i}^{(d)}}{b z_{i}^{(d)}}=b^{-2}<1
$$

and for $i \geqslant 1$

$$
\frac{b_{i}}{a_{i}}=\frac{b z_{i-1}^{(d)}}{b^{-1} z_{i}^{(d)}}<\frac{b^{2}}{b^{2}}=1 .
$$

Therefore, the sequences $a_{i}$ and $b_{i}$ interlace, i.e. $b_{d+1}<a_{d}<b_{d}<\ldots<b_{2}<a_{1}<b_{1}=0$. Since the sign of $b^{-d} P_{1}(z)+P_{2}(z)=K_{d+1}(z)$ at the endpoints of the interval $\left[a_{i}, b_{i}\right]$ are different, therefore we know for sure that there is a zero of $K_{d+1}(z)$ in the interval $\left[a_{i}, b_{i}\right]$ for any $1 \leqslant i \leqslant d$. To indicate a $d+1$ th zero of $K_{d+1}(z)$, observe that $P_{1}(z)$ and $P_{2}(z)$ has different sign on $\left(-\infty, b_{d+1}\right)$ and $P_{2}(z)$ has larger degree.

Since we found $d+1$ disjoint intervals containing at least one zero of $K_{d+1}(z)$, therefore each will contain exactly one zero, i.e., $K_{d+1}(z)$ has simple zeros $z_{0}^{(d+1)}>\cdots>z_{d+1}^{(d+1)}$.

To prove that the consecutive zeros are bounded, note that we have

$$
\frac{z_{i+1}^{(d+1)}}{z_{i}^{(d+1)}}>\frac{b_{i+1}}{a_{i}}=\frac{b z_{i}^{(d)}}{b^{-1} z_{i}^{(d)}}=b^{2} .
$$

Finally, note that $\left|z_{0}^{(d+1)}\right|<\left|a_{1}\right|=b^{-1}\left|z_{0}^{(d)}\right|$, which by induction is bounded by $b^{-d} z_{0}^{(1)}=b^{-d-1}$.

Remark 26. One could also easily obtain the real-rootedness of the polynomials $K_{d}(z)$ as follows. Let $P_{d}(z)=\sum_{i \geqslant 0} b^{-\left(\begin{array}{c}i \\ 2\end{array}\right)} z^{i}$. Then it is well known (see e.g. [7]) that $P_{d}(z)$ is a real rooted polynomial, therefore $P_{d}^{*}(z)=z^{d} P_{d}(1 / z)$ is also real rooted. Observe that the Schur-product of $P_{d}(z)$ and $P_{d}^{*}(z)$ is exactly $K_{d}(z)$, and since the Schur product preserves real-rootedness, we obtained that $K_{d}(z)$ has only real roots.

Now we have all the ingredients to prove the Theorem 1.

Proof of Theorem 1. By Lemma 23, we can express $Z_{L(G)}(z, b)$ as a specialization of the multivariate subgraph counting polynomial, where each key polynomial coincides with one of the members of $\left\{K_{d}(z) \mid d \geqslant 1\right\}$. By Lemma 25, we know each key polynomial has to be real rooted, i.e., each $K^{(v)}(z)$ has no roots in $\{z \in \mathbb{C}|| \arg (z) \mid<\pi\}$. Choosing $\alpha=0$ in Theorem 8 we obtain the desired statement. 
Next we prove Theorem 2 .

Proof of Theorem 2. Let $0<\alpha<\pi / 2$ be fixed and take $C=\{z \in \mathbb{C}|| \arg (z) \mid \geqslant \pi-\alpha\}$. Let us consider the following two variable polynomials

$$
K_{d}(z, t)=\sum_{i}\left(\begin{array}{l}
d \\
i
\end{array}\right) t^{\left(\begin{array}{c}
d-i \\
2
\end{array}\right)+\left(\begin{array}{l}
i \\
2
\end{array}\right)} z^{i}
$$

for some $1 \leqslant d \leqslant \Delta$.

We know from Lemma 25 that for any fixed $b \geqslant 1$, all the roots of $K_{d}\left(z, b^{-1}\right)$ are in $\operatorname{int}(C)$. By continuity we can find an open neighborhood $V_{b}$ of $b^{-1}$, such that $0 \notin V_{b}$ and for any $1 \leqslant d \leqslant \Delta$ and $\tau \in V_{b}$ the polynomial $K_{d}(z, \tau)$ has all its zeros contained in $\operatorname{int}(C)$. Let $U:=\cup_{b \geqslant 1} V_{b}^{-1}$. Then $U$ is an open set containing $[1, \infty)$ and for any $b^{\prime} \in U$ we have that the key polynomial $K_{d}\left(z, 1 / b^{\prime}\right)$ has no zeros contained in $\{z \in \mathbb{C}|| \arg (z) \mid<\pi-\alpha\}$.

Following the same argument as in the previous theorem, by the combination of Lemma 23 and Theorem 8, we obtain that for any $b^{\prime} \in U$ the polynomial $Z_{L(G)}\left(\lambda, b^{\prime}\right)$ has no root in $\{z \in \mathbb{C}|| \arg (z) \mid<\pi-2 \alpha\}$. In particular $Z_{L(G)}\left(1, b^{\prime}\right) \neq 0$ for all $b^{\prime} \in U$.

In a similar manner we can prove the following extensions:

Proposition 27. For any graph $G$ and values $b_{v} \geqslant 1$, a vector of variables $\lambda=\left(\lambda_{v}\right)_{v \in V}$, the multivariate polynomial

$$
F_{L(G)}\left(\lambda,\left(b_{v}\right)_{v \in V}\right):=\sum_{F \subseteq E} \prod_{v \in V} b_{v}^{-\left({ }_{G}^{d_{G}(v)-d_{F}(v)}\right)-\left({ }^{\left.d_{F}(v)\right)}\right)} \lambda_{v}^{d_{F}(v)}
$$

is weakly Hurwitz-stable, i.e. $\mathcal{S}[\pi / 2]$-nonvanishing.

Moreover, for any $\Delta \geqslant 2$ and $0<\alpha<\pi / 2$ there exists $a[1, \infty) \subseteq U_{\Delta, \alpha}$, such that the following holds: for any graph $G$ of maximum degree $\Delta$ and any $b_{v} \in U_{\Delta, \alpha}$, the polynomial

$$
F_{L(G)}\left(\lambda,\left(b_{v}\right)_{v \in V}\right) \neq 0
$$

if $\lambda_{v} \in\{z \in \mathbb{C}|| \arg (z) \mid<\pi-2 \alpha\}$.

\subsection{Disk around zero}

A priori, we know from [25, Remark 24], that for any graph $H$ of maximum degree at most $\Delta \geqslant 2$ the polynomial $Z_{H}(\lambda)$ has no root in a disk around 0 of radius for some $0<r<1 / b$. This is the last ingredient to conclude Corollary 3 .

For the sake of completeness and also as one more example for Wagner's theorem, we will describe a zero-free disk around zero for every fixed $b>1$. Let us denote by $\kappa_{d, b}$ the absolute value of the shortest zero of the key polynomial $K_{d}(z)$ from (4.1). Then:

Proposition 28. Let $G$ be a graph of maximum degree at most $\Delta \geqslant 2$, then

$$
Z_{L(G)}(z, b) \neq 0
$$

if $|z|<\kappa_{\Delta, b}^{2}$. 
Proof. From Lemma 25 we know that the polynomials $K_{d}(z)$ for $1 \leqslant d \leqslant \Delta$ are non-zero in a disk of radius $\kappa_{\Delta, b}$. The polynomial $(1+x y)$ is $\mathcal{D}$-nonvanishing, thus the corresponding base polynomial is also $\mathcal{D}$-nonvanishing. Thus by the second part of Theorem 8 , if none of the key polynomials vanishes in a disk of radius $\kappa_{\Delta, b}$, then the corresponding subgraph counting polynomial

$$
Z_{L(G)}(z, b)=b^{|E(L(G))|} Z_{\mathcal{W}}\left(G, 1,\left(b^{-\left({ }^{d_{G}(v)-i}\right)-\left(\begin{array}{c}
i \\
2
\end{array}\right)}\right)_{i=0}^{d_{G}(v)} ; z^{1 / 2}\right)
$$

will be zero free in a disk of radius $\kappa_{\Delta, b}^{2}$.

\section{$5 \quad$ Further remarks}

To finish the paper we would like to give two remarks.

One might wonder about the location of the zeros of the edge cover polynomials. First of all, if we look the closure of all possible zeros of $\mathcal{E}(\mathcal{H}, z)$, then we would obtain the whole complex plane, since already the closure of the zeros of domination polynomials are dense in $\mathbb{C}$ (see [8]). But what happens, if we consider zeros of the edge cover polynomial of graphs? For instance, the closure of zeros of the edge cover polynomial of paths is dense in $[-4,0]$ (see [11] ), therefore the described region in Theorem 5 is tight for real zeros.

Question 29. Is the set of zeros of edge-cover polynomials of graphs dense in the cardioid $\left\{-(1-\alpha)^{2}|| \alpha \mid \leqslant 1\right\} ?$

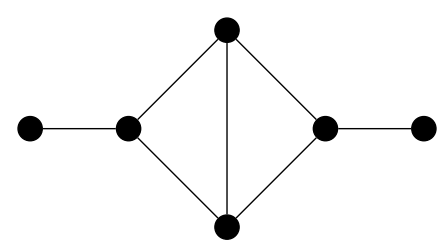

(a) A claw-free graph with $Z_{G}(\lambda, b)=$ $\lambda^{6}+\left(4 b^{3}+2 b\right) \lambda^{5}+\left(b^{6}+11 b^{4}+3 b^{2}\right) \lambda^{4}+$ $\left(12 b^{5}+8 b^{3}\right) \lambda^{3}+\left(b^{6}+11 b^{4}+3 b^{2}\right) \lambda^{2}+$ $\left(4 b^{3}+2 b\right) \lambda+1$

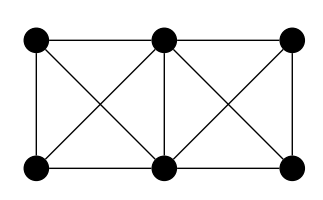

(b) A claw-free graph with $Z_{G}(\lambda, b)=$ $\lambda^{6}+\left(2 b^{5}+4 b^{3}\right) \lambda^{5}+\left(b^{8}+12 b^{6}+2 b^{4}\right) \lambda^{4}+$ $\left(16 b^{7}+4 b^{5}\right) \lambda^{3}+\left(b^{8}+12 b^{6}+2 b^{4}\right) \lambda^{2}+$ $\left(2 b^{5}+4 b^{3}\right) \lambda+1$

Figure 4: Claw-free graphs where $Z_{G}^{M C}(\lambda)=\lambda^{2}\left(1+\lambda^{2}\right)$ is not real-rooted.

Our second remark concerns the Ising model. Another famous anti-ferromagnetic model is the hard-core model, whose partition function is essentially the independence polynomial of graphs. It is well known that independence polynomials of line graphs have only real zeros [16]. Moreover, according to a theorem of Chudnovsky and Seymour [10], the independence polynomial has only real zeros also for claw-free graphs. Thus, the natural question whether the anti-ferromagnetic Ising model $Z_{G}(\lambda, b)$ has only real zeros for claw-free graphs for any choice of $b \geqslant 1$ arises. The answer to this question is no. To 
see why, let us fix a graph $G$, such that $Z_{G}(\lambda)$ is real rooted for any $b>1$. Also, denote by $M$ the size of a largest cut in the graph $G$. Then as $b \rightarrow \infty$

$$
\frac{Z_{G}(\lambda, b)}{b^{M}} \rightarrow \sum_{S \subseteq V} \lambda^{|S|} \mathbf{1}_{S} \text { realizes the maximum cut }=: Z_{G}^{M C}(\lambda)
$$

has to be a real rooted polynomial. For the claw-free graphs in Figure 4 this is not case.

\section{References}

[1] Saeed Akbari, Saeid Alikhani, Mohammad Reza Oboudi, and Yee-Hock Peng. On the zeros of domination polynomial of a graph. Combinatorics and Graphs, 531:109-115, 2010.

[2] Saieed Akbari and Mohammad Reza Oboudi. On the edge cover polynomial of a graph. European Journal of Combinatorics, 34(2):297-321, 2013.

[3] Saeid Alikhani and Yee-hock Peng. Introduction to domination polynomial of a graph. Ars. Comb., 114:257-266, 2014. arXiv:0905.2251.

[4] Alexander Barvinok. Combinatorics and complexity of partition functions, volume 9. Springer, 2016.

[5] Claude Berge. Hypergraphs, volume 45 of North-Holland Mathematical Library. North-Holland Publishing Co., Amsterdam, 1989. Combinatorics of finite sets, Translated from the French.

[6] Ivona Bezáková and William A Rummler. Sampling edge covers in 3-regular graphs. In International Symposium on Mathematical Foundations of Computer Science, pages 137-148. Springer, 2009.

[7] Jason Brown, Karl Dilcher, and Dante Manna. On the roots of expected independence polynomials. Journal of Graph Theory, 73, 072013.

[8] Jason I. Brown and Julia Tufts. On the roots of domination polynomials. Graphs and Combinatorics, 30(3):527-547, 2014.

[9] B. Chaluvaraju and V. Chaitra. Total domination polynomial of a graph. Journal of Informatics and Mathematical Sciences, 6(2):87-92, 2014.

[10] Maria Chudnovsky and Paul Seymour. The roots of the independence polynomial of a clawfree graph. Journal of Combinatorial Theory, Series B, 97(3):350-357, 2007.

[11] Péter Csikvári and Mohammad Reza Oboudi. On the roots of edge cover polynomials of graphs. European Journal of Combinatorics, 32(8):1407-1416, 2011.

[12] Markus Dod. Graph products of the trivariate total domination polynomial and related polynomials. Discrete Applied Mathematics, 209:92-101, 2016.

[13] Martin Dyer, Marc Heinrich, Mark Jerrum, and Haiko Müller. Polynomial-time approximation algorithms for the antiferromagnetic Ising model on line graphs. Combinatorics, Probability and Computing, pages 1-17, 2021. 
[14] M. E. Fisher. The nature of critical points. In W. E. Brittin, editor, Lecture notes in Theoretical Physics, volume 7c, pages 1-159. University of Colorado Press, 1965.

[15] Heng Guo, Jingcheng Liu, and Pinyan Lu. Zeros of ferromagnetic 2-spin systems. In Proceedings of the Fourteenth Annual ACM-SIAM Symposium on Discrete Algorithms, pages 181-192. SIAM, 2020.

[16] Ole J. Heilmann and Elliott H. Lieb. Theory of monomer-dimer systems. In Statistical Mechanics, pages 45-87. Springer, 1972.

[17] Lingxiao Huang, Pinyan Lu, and Chihao Zhang. Canonical paths for MCMC: from Art to Science. In Proceedings of the twenty-seventh annual ACM-SIAM symposium on Discrete algorithms, pages 514-527. SIAM, 2016.

[18] Nasrin Jafari and Saeid Alikhani. On the roots of total domination polynomial of graphs. Journal of Discrete Mathematical Sciences and Cryptography, 23(4):795-807, 2020.

[19] Tomer Kotek, James Preen, Frank Simon, Peter Tittmann, and Martin Trinks. Recurrence relations and splitting formulas for the domination polynomial. Electron. J. Combin., 19(3):\#P47, 2012.

[20] Chengyu Lin, Jingcheng Liu, and Pinyan Lu. A simple FPTAS for counting edge covers. In Proceedings of the twenty-fifth annual ACM-SIAM symposium on Discrete algorithms, pages 341-348. SIAM, 2014.

[21] Jingcheng Liu, Pinyan Lu, and Chihao Zhang. FPTAS for counting weighted edge covers. In European Symposium on Algorithms, pages 654-665. Springer, 2014.

[22] Jingcheng Liu, Alistair Sinclair, and Piyush Srivastava. The Ising partition function: Zeros and deterministic approximation. Journal of Statistical Physics, 174(2):287$315,2019$.

[23] Mohammad Reza Oboudi. On the roots of domination polynomial of graphs. Discrete Applied Mathematics, 205:126-131, 2016.

[24] Viresh Patel and Guus Regts. Deterministic polynomial-time approximation algorithms for partition functions and graph polynomials. SIAM Journal on Computing, 46(6):1893-1919, 2017.

[25] Han Peters and Guus Regts. Location of zeros for the partition function of the Ising model on bounded degree graphs. Journal of the London Mathematical Society, 101(2):765-785, 2020.

[26] Itiro Syôzi. Statistics of kagomé lattice. Progress of Theoretical Physics, 6(3):306-308, 1951.

[27] David G. Wagner. Weighted enumeration of spanning subgraphs with degree constraints. Journal of Combinatorial Theory, Series B, 99(2):347 - 357, 2009.

[28] Chen-Ning Yang and Tsung-Dao Lee. Statistical theory of equations of state and phase transitions. I. Theory of condensation. Physical Review, 87(3):404, 1952. 